\title{
Which children and young people are excluded from school? Findings from the Avon Longitudinal Study of Parents and Children (ALSPAC)
}

\author{
Amelia Paget, Claire Parker, William Henley, Jon Heron, Tamsin Ford, Alan Emond
}

Published Online February 26, 2015

Poster 31

Centre for Child and Adolescent Health, School of Social and Community Medicine, University of Bristol, Bristol, UK (A Paget MBChB, Prof A Emond MD); University of Exeter

Medical School, Exeter, UK (C Parker BSc, Prof W Henley PhD, ProfT Ford PhD); and School of Social and Community Medicine, University of Bristol, Bristol, UK (J Heron DPhil)

Correspondence to: Dr Amelia Paget, University of Bristol, Department of Child and Adolescent Health, University of Bristol, Oakfield House, Oakfield Grove, Clifton, Bristol BS8 2BN,

amelia.holme@bristol.ac.uk

\section{Abstract}

Background School exclusion is a disciplinary method used to remove a child from the school environment. It is known to affect certain groups disproportionately, including boys, some ethnic minorities, children in care, children in poverty, and children with special educational needs. Population-based studies on wider characteristics of excluded pupils are scarce. The aim of this study was to describe factors associated with school exclusion in the Avon Longitudinal Study of Parents and Children (ALSPAC), focussing on neurodevelopment and mental health.

Methods ALSPAC is a prospective population-based British birth cohort study, with the initial sample consisting of 14541 pregnancies. The study has data for whether a child has been permanently excluded from school up to the age of 8 years as reported by parents and also permanent and fixed period exclusions in the preceding 12 months as reported by parents and young people at age 16 years. Upstream risk factors were assessed for associations with exclusion on univariable analysis. The association with social communication difficulties was investigated with multivariable logistic regression.

Findings Data for exclusions up to the age of 8 years were available for 8245 ALSPAC participants and 4482 participants for exclusion at age 16 years. 53 pupils $(0 \cdot 6 \%)$ were excluded from school by age 8 years, and $390(8 \cdot 7 \%)$ at age 16 years. The odds of exclusion by 8 years and at 16 years were increased with male sex $(\mathrm{p}=0.001$ and $\mathrm{p}<0.0001$, respectively), low family income ( $\mathrm{p}=\mathbf{0} .014$ and $\mathrm{p}<0.0001)$, family adversity $(\mathrm{p}<0.0001$ for both), maternal psychopathology $(\mathrm{p}=\mathbf{0} .013$ and $\mathrm{p}=0.004)$, low intelligence quotient $(\mathrm{p}=0.041$ and $\mathrm{p}<0.0001)$, mental health difficulties $(\mathrm{p}<0.0001$ for both), psychiatric disorder ( $\mathrm{p}<\mathbf{0} .0001$ for both), social communication difficulties $(\mathrm{p}<\mathbf{0} 0001$ for both), antisocial activities $(p=0.004$ and $p<0.0001)$, bullying or being bullied $(p=0.005$ and $p<0.0001)$, low educational attainment $(p<0.0001$ for both), and increased special educational needs ( $p<0.0001$ for both). On multivariable analysis, having social communication difficulties above a clinical threshold on the Social Communication Disorders Checklist was strongly associated with exclusion by 8 years (odds ratio $7 \cdot 4,95 \%$ CI $3 \cdot 6-15 \cdot 4)$ and at 16 years $(2 \cdot 3,1 \cdot 5-3 \cdot 5)$, after adjustment for relevant confounders.

Interpretation Although cohort attrition and small numbers of exclusions at 8 years are limitations, this study suggests that school exclusion is associated with numerous risk factors identifiable at or before primary school entry. Child health professionals have an important role in the holistic assessment of children who are excluded, or who are at risk of school exclusion. There is particular need to ensure that mental health and neurodevelopmental difficulties are appropriately recognised and supported.

Funding National Institute for Health Research Academic Clinical Fellowship.

Contributors

TF devised the study. AP and CP did the statistical analysis, supervised by JH and WH. AP and CP wrote the abstract with input from AE and TF. All authors have seen and approved the final version of the abstract for publication.

Declaration of interests

We declare no competing interests. 